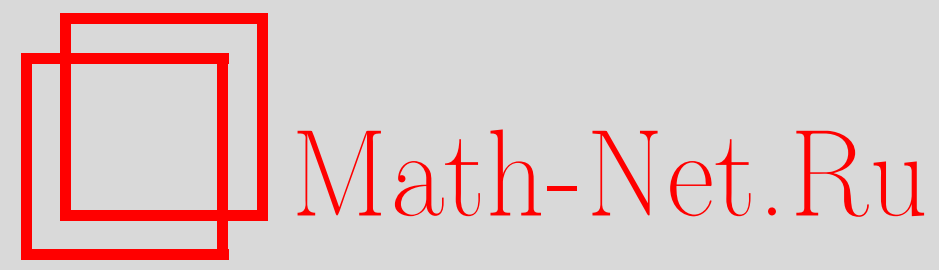

М. С. Тихов, Асимптотики статистических оценок по цензурированным выборкам для распределений с правильно меняющимися хвостами, Теория вероятн. и ее примен., 1997, том 42, выпуск 3, 531-552

DOI: https://doi.org/10.4213/tvp1951

Использование Общероссийского математического портала Math-Net.Ru подразумевает, что вы прочитали и согласны с пользовательским соглашением http://www . mathnet.ru/rus/agreement

Параметры загрузки:

IP: 34.239 .49 .27

26 апреля 2023 г., 05:56:06

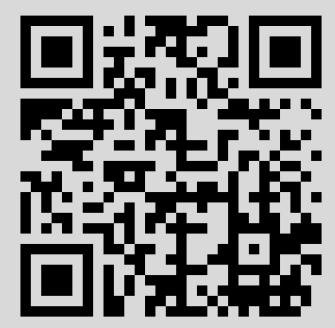




\section{АСИМПТОТИКИ СТАТИСТИЧЕСКИХ ОЩЕНОК ПО ЦЕНЗУРИРОВАННЫМ ВЫБОРКАМ ДЛЯ РАСПРЕДЕЛЕНИЙ С ПРАВИЛЬНО МЕНЯЮЩИМИСЯ ХВОСТАМИ}

В работе рассматривается асимптотическое поведение оценок Питмена $\hat{\theta}_{n}$ параметра сдвига плотности $f(x-\theta)=C(1+\alpha)(x-$ $\theta)^{\alpha} L(x-\theta), x \downarrow \theta, \alpha>-1, L(x)=1+D_{1}\left(1+\ell(1+\alpha)^{-1}\right) x^{\ell}+o\left(x^{\ell}\right)$, $\ell>0$, по наблюдениям за первыми $k$ порядковыми статистиками $\left(X_{n}^{(1)}, \ldots, X_{n}^{(k)}\right)$, когда $k=k(n) \rightarrow \infty, k / n \rightarrow 0$ при $n \rightarrow \infty$. Указаны предельные распределения $\hat{\theta}_{n}$ в зависимости от области значений параметра $\alpha$. В доказательстве используются свойства и асимптотические разложения гипергеометрических функций многих переменных. Приводятся простые асимптотически эффективные оценки параметра $\theta$ как линейные функции от наблюдаемых порядковых статистик.

Ключевые слова и фразы: цензурированные выборки, правильно меняющаяся плотность, параметр сдвига, параметрическое оценивание.

1. Введение. Пусть $\left\{X_{n}, n \geqslant 1\right\}$ - последовательность независимых одинаково распределенных случайных величин (с.в.), имеюших плотность распределения $f(x-\theta), \theta \in \mathbf{R}^{1}$, относительно меры Лебега на прямой с носителем на интервале $(\theta, \infty)$, причем $f(x)$ удовлетворяет условию:

$$
f(x)=C(1+\alpha) x^{\alpha} L(x) \quad \text { при } \quad x \downarrow 0,
$$

где $\alpha>-1$ и $C$ известны, $L(x)=1+D_{1}(1+\nu \ell) x^{\ell}+o\left(x^{\ell}\right), \ell>0$, $\nu=1 /(1+\alpha), L(x)$ - медленно меняющаяся функция. Пусть $\left\{X_{n}^{(i)}\right.$, $1 \leqslant i \leqslant n\}$ - вариационный ряд (в.р.), $X_{n}^{(i)}=X_{n}^{(i)}\left(X_{1}, X_{2}, \ldots, X_{n}\right)$ есть $i$-я порядковая статистика, а $X_{n}^{(1, k)}=\left(X_{n}^{(1)}, X_{n}^{(2)}, \ldots, X_{n}^{(k)}\right)$ есть отрезок вариационното ряда. Цель статьи - исследовать поведение оценок Питмена $\hat{\theta}_{n}=\hat{\theta}_{n}\left(X_{n}^{(1, k)}\right)$ параметра сдвига $\theta$ плотности $f(x-\theta)$ со свойством (1), построенных по отрезку в.р. $X_{n}^{(1, k)}$, когда $k=k(n) \rightarrow \infty$, но $k / n \rightarrow 0$ при $n \rightarrow \infty$. Так как заменами $Y=-X$ или $Z=1 / X$ можно переносить

*Нижегородский университет им. Н. И. Лобачевского, кафедра прикладной теории вероятностей, пр. Гагарина, 23, 603600 Нижний Новгород, Россия. 
результаты, найденные для отрезка в.p. $X_{n}^{(1, k)}$ на результаты для отрезка $X_{n}^{(n-k+1, n)}=\left(X_{n}^{(n-k+1)}, \ldots, X_{n}^{(n)}\right)$, и плотности $f_{Y}(x)=f(-x)$ или $f_{Z}(x)=x^{-2} f\left(x^{-1}\right)$, то мы будем рассматривать только отрезок $X_{n}^{(1, k)}$ и плотность $f(x)$.

Асимптотическое поведение статистик, построенных как по отрезку $X_{n}^{(1, k)}$, так и по $X_{n}^{(n-k+1, n)}$, рассматривали Р. Дэвис (R. Davis) и С. Резник (S. Resnik) [18], П. Холл (P. Hall) и А. Уэлш (A. Welsh) [20], С. Чёргё (S. Csörgö) [17], М. Тихов [15]. В применении к распределению (1) исследовался в основном случай $\alpha>1$. В [19] П. Холл (Р. Hall) изучил асимптотическое поведение оценок максимального правдоподобия (ОМП) параметра сдвига $\theta$ плотности $f_{1}(\theta-x), f_{1}(x)=C(1+\alpha) x^{\alpha}\left(1+O\left(x^{\ell}\right)\right)$, $x \downarrow 0$, где $\alpha \geqslant 0, \ell>0$. В частности, он доказал асимптотическую нормальность ОМП параметра $\theta$, построенных по отрезку в.р. $X_{n}^{(n-k+1, n)}$, когда $\alpha \geqslant 1$, при известных $C$ и $\alpha$, а также асимптотическую нормальность ОМП параметров $\alpha$ и $\theta$. Его доказательства основывались на представлении A. Реньи (A. Rényi) [21] для порядковых статистик из показательного распределения.

В данной статье изучается асимптотическое поведение оценок Питмена $\hat{\theta}_{n}$ при известных $C$ и $\alpha$ на базе метода И. А. Ибрагимова и Р. 3. Хасьминского [6]. Для применения этого метода сначала устанавливается локальное асимптотическое поведение отношения правдоподобия (OII) (теорема 1) подходом, разработанным в работах [12]-[14], на основании чего показывается, что имеет место указанное в теореме 2 предельное распределение оценок Питмена. Локальное асимптотическое поведение ОП в случае плотности (1) выводится исходя из свойств и асимптотических разложений гипергеометрических функций оцной и многих переменных. Это позволяет охватить также значения $\alpha \in(-1,0) \cup(0,1)$. Для случаев $\alpha \geqslant 1$ и $\alpha=0$ в п. 5 приводятся простые оценки параметра сдвига $\theta$ и доказывается их асимптотическая эффективность, когда $f(x)=(1+\alpha) x^{\alpha}, 0<x<1$. В случае $0<|\alpha|<1$ сравниваются две оценки параметра сдвига $\theta$, порядок сходимости которых такой же, как и у оценки Питмена. Подсчитано, что для $\alpha$, больших примерно 0.205 , первая из них лучше, чем вторая, а при $\alpha$, меньших - хуже в смысле предельной дисперсии.

2. Обозначения. Положим

$$
\begin{aligned}
& \lambda_{n}=(C n)^{-\nu} L^{*}\left(\frac{1}{n C}\right), \quad b_{n}=\left(\frac{k}{n C}\right)^{\nu} L^{*}\left(\frac{k}{n C}\right) \\
& a_{n}=b_{n} \nu k^{-1 / 2}, \quad z=a_{n} x_{2}+b_{n}, \quad \beta_{n}=\frac{1}{\mu(n)}
\end{aligned}
$$

$L^{*}(x)=1-D_{1} \nu x^{\ell}+o\left(x^{\ell}\right)$ есть сопряженная с $L(x)=1+D_{1} x^{\ell}+o\left(x^{\ell}\right)$ медленно меняющаяся функция $[10$, с. 31$], \nu=1 /(1+\alpha), \mu(n)-$ нормируюший множитель, вид которого определяется областью значений 
параметра $\alpha$ (см. теорему 1).

Если $F(x)$ - функция распределения (ф.р.) с плотностью распределения (п.р.) $f(x)$, удовлетворяющей условию (1), то $F(x)$ удовлетворяет условию

$$
F(x)=C x^{1+\alpha} L_{1}(x) \quad \text { при } \quad x \downarrow 0 .
$$

Обьектом нашего исследования будет случайная функция

$$
\begin{aligned}
\xi_{n}(u)= & \ln f_{n}\left(X_{n}^{(1)}-\frac{u}{\mu(n)}, \ldots, X_{n}^{(k)}-\frac{u}{\mu(n)}\right) \\
& -\ln f_{n}\left(X_{n}^{(1)}, \ldots, X_{n}^{(k)}\right), \quad u \in \mathbf{R}^{1},
\end{aligned}
$$

где

$$
f_{n}\left(y_{1}, \ldots, y_{k}\right)= \begin{cases}(n-k+1)_{k} \prod_{i=1}^{k} f\left(y_{i}\right)\left(1-F\left(y_{k}\right)\right)^{n-k} \\ \text { если } y_{1}<\cdots<y_{k} \\ \text { в противном случае }\end{cases}
$$

есть плотность распределения вектора $X_{n}^{(1, k)},(n-k+1)_{k}=\Gamma(n+1) / \Gamma(n-$ $k+1)$ есть символ Похгаммера $(k=1,2, \ldots, n), \Gamma(\alpha)$ - гамма-функция Эйлера, а также оченки Питмена

$$
\hat{\theta}_{n}=\frac{\int_{-\infty}^{\infty} \theta f_{n}\left(X_{n}^{(1)}-\theta, \ldots, X_{n}^{(k)}-\theta\right) d \theta}{\int_{-\infty}^{\infty} f_{n}\left(X_{n}^{(1)}-\theta, \ldots, X_{n}^{(k)}-\theta\right) d \theta}
$$

параметра сдвига $\theta$.

Мы подберем нормирующий множитель $\mu(n)$ таким, чтобы предельные распределения $\mu(n)\left(\hat{\theta}_{n}-\theta\right)$ при $n \rightarrow \infty$ были бы невырожденными.

Определим функции:

$$
\begin{aligned}
\psi_{n}(x, u) & =\ln f\left(x-\frac{u}{\mu(n)}\right)-\ln f(x), \\
M_{n}(x, u) & =\ln \left(1-F\left(x-\frac{u}{\mu(n)}\right)-\ln (1-F(x)),\right. \\
V_{n}(\mathbf{t} ; x) & =\exp \left\{i \sum_{j=1}^{m} t_{j} \psi_{n}\left(x, u_{j}\right)\right\}, \quad \mathbf{t}=\left(t_{1}, \ldots, t_{m}\right), \\
I_{n}(\mathbf{t} ; z) & =\int_{-\infty}^{z} V_{n}(\mathbf{t} ; x) f(x) d x, \quad U_{n}(\mathbf{t} ; z)=\frac{I_{n}(\mathbf{t} ; z)}{F(z)} .
\end{aligned}
$$

Положим $t_{+}=\sum_{j=1}^{m} t_{j}$, и пусть $\varphi_{n}(\mathrm{t})=\mathbf{E}\left(\exp \left\{i \sum_{j=1}^{m} \xi_{n}\left(u_{j}\right) t_{j}\right\}\right)$ есть характеристическая функция вектора $\left(\xi_{n}\left(u_{1}\right), \ldots, \xi_{n}\left(u_{m}\right)\right)$, где символ $\mathbf{E}_{\theta}(\cdot)$ обозначает математическое ожидание по распределению $F(x-$ $\theta), \mathbf{E}(\cdot)=\mathbf{E}_{0}(\cdot)$. 
Через $\eta(\alpha)$ будем обозначать случайную величину, имеющую распределение Вейбулла с ф.р. $G_{\alpha}(x)=1-\exp \left(-x^{1+\alpha}\right), x>0$, полагая $\eta(0)=\eta$; через $\zeta$ - с.в., имеюшую стандартное нормальное распределение $\mathcal{N}(0,1)$ с ф.р. $\Phi(x)=\int_{-\infty}^{x}(2 \pi)^{-1 / 2} \exp \left(-x^{2} / 2\right) d x$, а через $N\left(0, \sigma^{2}\right)-$ с.в., имеющую ф.p. $\Phi(x / \sigma)$.

Нам понадобится гипергеометрическая функция многих переменных Лауричелла $F_{1}\left(a, b_{1}, \ldots, b_{m} ; c ; x_{1}, \ldots, x_{m}\right)$, которая определяется в виде интеграла (см. [9, с. $452,7.2 .4 .57])$ :

$$
\begin{aligned}
& F_{1}\left(a, b_{1}, \ldots, b_{m} ; c ; x_{1}, \ldots, x_{m}\right) \\
& \quad=\frac{\Gamma(c)}{\Gamma(a) \Gamma(c-a)} \int_{0}^{1} u^{a-1}(1-u)^{c-a-1}\left(1-u x_{1}\right)^{-b_{1}} \cdots\left(1-u x_{m}\right)^{-b_{m}} d u,
\end{aligned}
$$

$\operatorname{Re} c>\operatorname{Re} a>0,\left|\arg \left(1-x_{i}\right)\right|<\pi, i=1,2, \ldots, m$, а также в виде сходящегося ряда (см. $[9$, с. $449,7.2 .4 .15])$ :

$$
\begin{aligned}
& F_{1}\left(a, b_{1}, \ldots, b_{m} ; c ; x_{1}, \ldots, x_{m}\right) \\
& \quad=\sum_{s_{1}, \ldots, s_{m} \geqslant 0} \frac{(a)_{s_{1}}+\cdots+s_{m}\left(b_{1}\right)_{s_{1}} \cdots\left(b_{m}\right) s_{m}}{(c)_{s_{1}+\cdots+s_{m}}} \frac{x_{1}^{s_{1}}}{s_{1} !} \cdots \frac{x_{m}^{s_{m}}}{s_{m} !}
\end{aligned}
$$

$\left|x_{i}\right|<1, i=1,2, \ldots, m$. Пусть, кроме того,

$$
\varphi_{x}(t, u)=\exp \left\{x^{1+\alpha}\left(1-F_{1}\left(-i t \alpha,-\alpha-1 ;-\alpha ; \frac{u}{x}\right)\right)\right\},
$$

если $u<x$, цля $t \in \mathbf{R}^{1}, \alpha \in(-1,0) \cup(0,1)$, где $F_{1}\left(a, b_{1} ; c ; x\right)$ в случае одного переменного есть гипергеометрическая функция Гаусса (см. $[2$, $2.1 .1(2)$, c. $69 ; 2.1 .3(10)$, c. 72$])$.

Пусть

$$
\left.J_{n}(\theta)=\mathrm{E}_{\theta}\left(\frac{\partial}{\partial \theta}\right) \ln f_{n}\left(X_{n}^{(1)}-\theta, \ldots, X_{n}^{(k)}-\theta\right)\right)^{2}
$$

есть количество информации, содержапееся в отрезке в.р. $X_{n}^{(1, k)}$.

3. Результаты. Основной результат статьи составляют следующие теоремы.

Теорема 1. Пусть плотность $f(x)$ удовлетворяет условию (1). Тогда конечномерные распределения случайных функчий $\left\{\xi_{n}(u), u \in\right.$ $\mathbf{R}^{1}$ \} сходятся при $n \rightarrow \infty \propto$ конечномерным распределениям функиии $\left\{\xi(u), u \in \mathbf{R}^{1}\right\}$, причем

(i) ecлu $(U 1): \alpha>1, \mu(n)=\nu^{-1} C^{\nu} n^{\nu} k^{1 / 2-\nu} / L^{*}(k / n), k=k(n) \rightarrow \infty$ $u k=o\left(n^{r /(r+1 / 2)}\right)$, гдe $r=\min (\nu \ell, 1)$ npu $L(x) \neq 1, r=1$ npu $L(x)=1$, mo

$$
\xi(u)=-\frac{1}{2} u^{2} \alpha^{2}\left(\alpha^{2}-1\right)^{-1}+u \alpha\left(\alpha^{2}-1\right)^{-1 / 2} \zeta
$$


(ii) ecsu $(U 2): \alpha=1, \mu(n)=(C n \ln k)^{1 / 2} / L^{*}(k / n), k^{(r+1 / 2) / r} n^{-1}$ $\times(\ln k)^{-1 / 2} \rightarrow 0, m o$

$$
\xi(u)=-\frac{1}{2} u^{2}+u \zeta
$$

(iii) $е с л u(U 3): \alpha=0 u \mu(n)=C n, m o$

$$
\xi(u)=\left\{\begin{array}{lll}
u & \partial \Omega g & u \leqslant \eta \\
-\infty & \partial \Omega A & u>\eta
\end{array}\right.
$$

(iv) если (U4): $0<|\alpha|<1, \mu(n)=C^{\nu} n^{\nu}$, то последовательность характеристических функиий $\varphi_{n}(t)=\mathrm{E}\left(\exp \left(i t \xi_{n}(u)\right)\right)$ сходится $\kappa$ функчuน

$$
(1+\alpha) \int_{u}^{\infty} x^{\alpha} \exp \left\{-x^{1+\alpha} F_{1}\left(-i t \alpha,-\alpha-1 ;-\alpha ; u x^{-1}\right)\right\} d x
$$

Теорема 2. Пусть $\hat{\theta}_{n}$ - оценка Питмена параметра сдвига распределения $F(x-\theta)$, построенная по отрезку ө.p. $X_{n}^{(1, k)}, k=k(n) \rightarrow \infty$, $k / n \rightarrow 0, n \rightarrow \infty$, где $F(x)$ удовлетовряет условию (2).

(i) Если $\alpha>1$ и выполнено условие (U1), то

$$
n^{\nu} k^{1 / 2-\nu}\left(\hat{\theta}_{n}-\theta\right) \underset{n \rightarrow \infty}{\stackrel{d}{\longrightarrow}} N\left(0,(1-2 \nu) \nu^{2}(1-\nu)^{-2} C^{-2 \nu}\right)
$$

(ii) если $\alpha=1$ и выполнено условие (U2), то

$$
(n \ln k)^{1 / 2}\left(\hat{\theta}_{n}-\theta\right) \underset{n \rightarrow \infty}{\stackrel{d}{\longrightarrow}} N\left(0, C^{-1}\right)
$$

(iii) ecлu $\alpha=0, m o$

$$
C n\left(\hat{\theta}_{n}-\theta\right) \underset{n \rightarrow \infty}{\stackrel{d}{\longrightarrow}} \eta-1 .
$$

Теорема 3. Пусть плотность $f(x)$ удовлетворяет условию (1). Тогда для мюбой последовательности оченок $\left\{T_{n}\right\}$ параметра сдвига плотности $f(x-\theta)$ и любого $\beta>0$ имеют место следующие утверждения:

(i) при условии (U1)

$\varliminf_{\delta \rightarrow 0} \varliminf_{n \rightarrow \infty} \sup _{|\theta-t|<\delta} \mathbf{E}_{t}\left|\mu(n)\left(T_{n}-t\right)\right|^{\beta} \geqslant \frac{(2(1-2 \nu))^{\beta / 2} \Gamma((\beta+1) / 2)}{(1-\nu)^{\beta} \Gamma\left(\frac{1}{2}\right)} ;$

(ii) при условии (U2)

$$
\varliminf_{\delta \rightarrow 0} \varliminf_{n \rightarrow \infty} \sup _{|\theta-t|<\delta} \mathbf{E}_{t}\left|\mu(n)\left(T_{n}-t\right)\right|^{\beta} \geqslant 2^{\beta / 2} \frac{\Gamma((\beta+1) / 2)}{\Gamma\left(\frac{1}{2}\right)} ;
$$


(iii) при условии (U3)

$$
\varliminf_{\delta \rightarrow 0} \varliminf_{n \rightarrow \infty} \sup _{|\theta-t|<\delta} \mathbf{E}_{t}\left|\mu(n)\left(T_{n}-t\right)\right|^{\beta} \geqslant \min _{u} \int_{0}^{\infty}|y-u|^{\beta} e^{-y} d y .
$$

В теореме 1 определено локальное асимптотическое поведение отношения правдоподобия, в теореме 2 указаны предельные распределения оценок Питмена. Теорема 3 дает асимптотически минимаксные границы рисков статистических оценок.

4. Доказательства. Установим сначала асимптотическое поведение порядковых статистик $X_{n}^{(k)}$ и $X_{n}^{(1)}$.

Лемма 1. Пусть б.p. $F(x)=C x^{1+\alpha} L(x), L(x)-$ произвольная медленно меняющаяся в нуле функция, а $L^{*}(x)$ - сопряженная с $L(x)$ медленно меняюцаяся функиия. Неравенство $L(x) \neq 1$ ' понимается в том смысле, ито $L(x)$ не равна единиче из некоторой охрестности нуля

$$
\lambda_{n}=(C n)^{-\nu} L^{*}\left(\frac{1}{n C}\right), \quad b_{n}=\left(\frac{k}{n C}\right)^{\nu} L^{*}\left(\frac{k}{n C}\right), \quad a_{n}=b_{n} \nu k^{-1 / 2} .
$$

(i) Ec.ли $k \rightarrow \infty, k / n \rightarrow 0$ nрu $n \rightarrow \infty$, mo

$$
\mathbf{P}\left\{\frac{X_{n}^{(k)}-b_{n}}{a_{n}}<x\right\} \rightarrow \Phi(x)
$$

(ii) $\operatorname{Eсли~} k=1$, mo

$$
P\left\{X_{n}^{(1)}<x \lambda_{n}\right\} \longrightarrow G_{\alpha}(x)
$$

Д ок а 3 а т е ль с т в о. Рассмотрим часть (i) леммы. Как известно (см. $[11$, с. 254 , теорема 2]), для сходимости (3) необходимо и достаточно, чтобы при любых $x \in \mathbf{R}^{1}$

$$
\frac{n F\left(a_{n} x+b_{n}\right)-k}{\sqrt{k}} \rightarrow x, \quad n \rightarrow \infty .
$$

Из определения коэффициентов $a_{n}$ и $b_{n}$ для $F(x)=C x^{1+\alpha} L(x)$ имеем:

$$
n F\left(a_{n} x+b_{n}\right)=k\left(1+\frac{x \nu}{\sqrt{k}}\right)^{1+\alpha} \delta_{n}
$$

где

$$
\delta_{n}=\left(L^{*}\left(\frac{k}{n C}\right) L^{\nu}\left(\left(\frac{k}{n C}\right)^{\nu}\left(1+\frac{x \nu}{\sqrt{k}}\right)\right) L^{*}\left(\frac{k}{n C}\right)\right)^{1+\alpha}
$$

Известно [см. 10, с. 30, теорема 1.5], что если $L(x)$ и $L^{*}(x)-$ сопряженные медленно меняющиеся функции, то $L^{*}(x) L\left(x L^{*}(x)\right) \longrightarrow 1$ при 
$x \rightarrow \infty$, поэтому $\delta_{n} \rightarrow 1, n \rightarrow \infty$, равномерно по $|x| \leqslant C_{2}<\infty$. Значит, при $n \rightarrow \infty$ равномерно по $|x| \leqslant C_{2}$ выполняется $n F\left(a_{n} x+b_{n}\right) \sim$ $k(1+x / \sqrt{k}+o(1 / \sqrt{k}))$, поэтому соотношение $(5)$, а за ним и $(3)$, будут иметь место.

Перейдем к части (ii). Из определения правильно меняющихся функций $F(\tau x) / F(x) \rightarrow \tau^{1+\alpha}(x \downarrow 0, \tau>0)$. По теореме 6 из $[11$, с. 197] необходимым и достаточным условием принадлежности $F(x)$ области притяжения закона $G_{\alpha}(x)$ является выполнение условия $n F\left(\lambda_{n} x\right) \longrightarrow x^{1+\alpha}$, $n \rightarrow \infty$. Для доказательства последнего необходимо использовать лемму 1.8 из $\left[10\right.$, с. 29], согласно которой если $F(x)=C x^{1+\alpha} L(x)$ при $x \downarrow 0$, то единственной асимптотически обратной к $F(x)$ функцией будет $R^{*}(x)=(x / C)^{\nu} L^{*}(x / C)$, что доказывает (4).

Доказате льс т в о те оремы 1. Мы будем доказывать сходимость характеристических функций (х.ф.) $\varphi_{n}(\mathrm{t})$ к предельной х.ф. $\varphi(\mathbf{t})$, откуда будет следовать сходимость конечномерных распределений функций $\left\{\xi_{n}(u), u \in \mathbf{R}^{1}\right\}$ к распределениям случайной функции $\left\{\xi(u), u \in \mathbf{R}^{1}\right\}$.

Пользуясь свойствами порядковых статистик, получаем

$$
\begin{aligned}
\varphi_{n}(\mathbf{t}) & =\mathbf{E}\left(\mathbf{E}\left(\exp \left\{i \sum_{j=1}^{m} t_{j} \xi_{n}\left(u_{j}\right)\right\} \mid X_{n}^{(1)}, X_{n}^{(k)}\right)\right) \\
& =\mathbf{E}\left(Q_{n}\left(\mathbf{t} ; X_{n}^{(1)}, X_{n}^{(k)}\right)\right) \\
& =\iint_{y<z} Q_{n}(\mathbf{t} ; y, z) p_{n}^{(1, k)}(y, z) d y d z \quad\left(\mathbf{t} \in \mathbf{R}^{m}\right),
\end{aligned}
$$

где

$$
\begin{aligned}
Q_{n}(\mathbf{t} ; y, z)= & \frac{\int_{y}^{z} V_{n}(\mathbf{t} ; x) f(x) d x}{(F(z)-F(y))^{k-2}} V_{n}(\mathbf{t} ; y) V_{n}(\mathbf{t} ; z) \\
& \times \exp \left\{i(n-k) \sum_{j=1}^{m} t_{j} M_{n}\left(z, u_{j}\right)\right\}, \\
p_{n}^{(1, k)}(y, z)= & \frac{\Gamma(n+1)}{\Gamma(n-k+1) \Gamma(k-1)} \\
& \times(F(z)-F(y))^{k-2}(1-F(z))^{n-k} f(y) f(z)
\end{aligned}
$$

для $y<z$, и $p_{n}^{(1, k)}(y, z)=0$ в противном случае.

Сделаем в (6) замену переменных: $z=a_{n} x_{2}+b_{n}, y=\lambda_{n} x_{1}$. Тогда

$$
\begin{aligned}
\varphi_{n}(\mathbf{t})= & \iint_{\lambda_{n} x_{1}<a_{n} x_{2}+b_{n}} Q_{n}\left(\mathrm{t} ; \lambda_{n} x_{1}, a_{n} x_{2}+b_{n}\right) \\
& \times a_{n} \lambda_{n} p^{(1, k)}\left(\lambda_{n} x_{1}, a_{n} x_{2}+b_{n}\right) d x_{1} d x_{2} .
\end{aligned}
$$


Известно (см. $\left[11\right.$, с. 46, 53]), что $a_{n} \lambda_{n} p_{n}^{(1, k)}\left(\lambda_{n} x_{1}, a_{n} x_{2}+b_{n}\right)$ сходится при $n \rightarrow \infty$ к плотности $h_{1}\left(x_{1}\right) h_{2}\left(x_{2}\right)$, где $h_{1}(x)=(1+\alpha) x^{\alpha} \exp \left(-x^{1+\alpha}\right)$, для $x>0 ; h_{2}(x)=(2 \pi)^{-1 / 2} \exp \left(-x^{2} / 2\right)$; причем эта сходимость равномерна по $\left(x_{1}, x_{2}\right) \in B$, где $B=\left\{\left(x_{1}, x_{2}\right): 0<x_{1} \leqslant C_{1},\left|x_{2}\right| \leqslant C_{2}\right\}$.

Из определения функции $Q_{n}(\mathbf{t} ; y, z)$ следует, что $\left|Q_{n}(\mathbf{t} ; y, z)\right| \leqslant 1$ для лғобых $y, z$. Кроме того, $\mathbf{P}\left\{X^{(1)}>\lambda_{n} C_{1},\left|X_{n}^{(k)}-b_{n}\right|>a_{n} C_{2}\right\} \leqslant \mathbf{P}\left\{\mid X_{n}^{(k)}-\right.$ $\left.b_{n} \mid>a_{n} C_{2}\right\}$. Поэтому (см. [15, с. 662]) можно выбрать $C_{2}>0$ так, чтобы для заданного $\varepsilon>0$ выполнялись неравенства: $\varepsilon C_{2}^{2}>2$ и $\mathbf{P}\left\{\left|X_{n}^{(k)}-b_{n}\right|>\right.$ $\left.a_{n} C_{2}\right\}<\varepsilon$.

В таком случае из работы [13] получаем, что достаточно найти предел при $n \rightarrow \infty$ функции $Q_{n}\left(\mathrm{t} ; \lambda_{n} x_{1}, a_{n} x_{2}+b_{n}\right)$ для $\left(x_{1}, x_{2}\right) \in B$, к чему мы сейчас и перейдем.

Пусть $u<0$. Заметим, что $F\left(\lambda_{n} x_{1}\right) / F\left(a_{n} x_{2}+b_{n}\right) \sim O(1 / k)$ и $V_{n}(\mathbf{t} ; y)=1+o(1), V_{n}(\mathbf{t} ; z)=1+o(1)$ равномерно на множестве $B$, поэтому при $n \rightarrow \infty$

$$
\begin{aligned}
& Q_{n}\left(\mathbf{t} ; \lambda_{n} x_{1}, a_{n} x_{2}+b_{n}\right) \\
& \quad=U_{n}^{k}\left(\mathbf{t}, a_{n} x_{2}+b_{n}\right) \exp \left\{i(n-k) \sum_{j=1}^{m} t_{j} M_{n}\left(a_{n} x_{2}+b_{n}, u_{j}\right)\right\}(1+o(1)) .
\end{aligned}
$$

Так как $U_{n}(\mathbf{t} ; x)=I_{n}(\mathbf{t} ; x) / F(x)$, то рассмотрим функщию

$$
I_{n}(\mathbf{t} ; z)=\int_{0}^{z} C(1+\alpha) x^{\alpha} g(x) L(x) d x
$$

где $g(x)=\prod_{j=1}^{m}\left(1-\beta_{n} u_{j} / x\right)^{i t_{j} \alpha}$.

Заменим в интеграле (7) переменную $x$ на $z v$. В таком случае

$$
I_{n}(\mathbf{t} ; z)=C(1+\alpha) z^{\alpha+1} \int_{0}^{1} v^{\alpha} g(z v) L(z v) d v
$$

Ясно, что $|g(z v)| \leqslant 1$. Пусть $A(z)=\int_{0}^{1} v^{\alpha}(L(z v) / L(z)-1) d v$. По теореме о равномерной сходимости для медленно меняющихся функций (см. $[10$, с. 10]) $A(z) \rightarrow 0$ при $z \rightarrow 0$. Значит, при $z \rightarrow 0$

$$
\begin{aligned}
\int_{0}^{1} v^{\alpha} g(z v) L(z v) d v & \sim L(z) \int_{0}^{1} v^{\alpha} g(z v) d v \\
U_{n}(\mathbf{t} ; z) & \sim(1+\alpha) \int_{0}^{1} v^{\alpha} g(z v) d v
\end{aligned}
$$

поэтому в дальнейшем будем обозначать $U_{n}(\mathbf{t} ; z)=(1+\alpha) \int_{0}^{1} v^{\alpha} g(z v) d v$.

С л у ч а й (i). Используя интегральное представление гипергеометрической функции Лауричелла, запишем функцию $U_{n}(\mathbf{t} ; z)$ в следующем виде: 


$$
\begin{aligned}
U_{n}(\mathbf{t} ; z)= & \frac{1+\alpha}{\alpha+1-i \alpha t_{+}} \prod_{j=1}^{m}\left(-\frac{u_{j} \beta_{n}}{z}\right)^{i \alpha t_{j}} \\
& \times F_{1}\left(\alpha+1-i \alpha t_{+},-i \alpha t_{1}, \ldots,-i \alpha t_{m} ; \alpha+2-i \alpha t_{+}\right. \\
& \left.\frac{z}{\beta_{n} u_{1}}, \ldots, \frac{z}{\beta_{n} u_{m}}\right)
\end{aligned}
$$

поскольку $\operatorname{Re}\left(\alpha+2-i \alpha t_{+}\right)>\operatorname{Re}\left(\alpha+1-i \alpha t_{+}\right)>0$.

Пусть для начала $m=1$. Тогда $F_{1}$ есть гипергеометрическая функция Гаусса одной переменной. Из формулы 2.10.(2) [2, с. 116] для аналитического продолжения гипергеометрического ряда получаем (если $\alpha \neq 2,3, \ldots$ и $\left.\beta_{n}=a_{n}\right)$

$$
\begin{aligned}
U_{n}(t ; z)= & -\left(\frac{a_{n} u}{z}\right)^{1+\alpha} \frac{\Gamma(\alpha+1-i t \alpha) \Gamma(-\alpha)}{\Gamma(-i t \alpha)} \\
& +F_{1}\left(-i t \alpha,-\alpha-1 ;-\alpha ; \frac{a_{n} u}{z}\right)
\end{aligned}
$$

где

$$
F_{1}\left(-i t \alpha,-\alpha-1 ;-\alpha ; \frac{a_{n} u}{z}\right)=1+\frac{i t a_{n} u}{\nu z}+\frac{i t \alpha(1-i t \alpha) a_{n}^{2} u^{2}}{2 \nu(1-\alpha) z^{2}}+o\left(\frac{1}{k}\right)
$$

Так как $\alpha+1>2$, то в (9) первое слагаемое будет порядка $o(1 / k)$. Из (9) и (10) теперь нетрудно вывести, что

$$
U_{n}(t ; z)=1-\frac{i t u}{\sqrt{k}}+\frac{i t x_{2} u}{k(1+\alpha)}-\frac{i \alpha t(i t \alpha-1) u^{2}}{2\left(\alpha^{2}-1\right) k}+o\left(\frac{1}{k}\right)
$$

В то же время при $n \rightarrow \infty$

$$
\begin{aligned}
M_{n}(z, u)= & \frac{u \sqrt{k}}{n}+\frac{\left(2 x_{2}-u\right) u \alpha}{2 n(1+\alpha)}+o\left(\frac{1}{n}\right) \\
& +O\left(\frac{k^{\nu \ell+1 / 2}}{n^{\nu \ell+1}}\right)+O\left(\frac{k^{3 / 2}}{n^{2}}\right)
\end{aligned}
$$

Из условия $(U 1)$ заключаем, что $O\left(k^{\nu \ell+1 / 2} / n^{\nu \ell+1}\right)+O\left(k^{3 / 2} / n^{2}\right)=o(1 / n)$.

Тогда при $n \rightarrow \infty$

$$
Q_{n}\left(t ; \lambda_{n} x_{1}, a_{n} x_{2}+b_{n}\right) \longrightarrow \exp \left\{i t u x_{2}+\left(-D+t^{2}-\frac{i t \alpha}{1+\alpha}\right) \frac{u^{2}}{2}\right\}
$$

где $D(t)=t^{2} \alpha^{2}\left(\alpha^{2}-1\right)^{-1}+i t \alpha^{2}\left(\alpha^{2}-1\right)^{-1}$. 
Если же $\alpha$ равно целому числу (в нашем случае $2,3, \ldots$ ), то мы воспользуемся разложением гипергеометрической функции Гаусса вида 2.10.(9) [2, с. 117], согласно которому

$$
\begin{aligned}
U_{n}(t ; z)= & (-1)^{\alpha}\left(-\frac{a_{n} u}{z}\right)^{1+\alpha} \sum_{j=1}^{\infty} \frac{\Gamma(\alpha+1-i t \alpha)}{j \Gamma(-i t \alpha) \Gamma(\alpha+1+j)}\left(\frac{a_{n} u}{z}\right)^{j} \\
& +\sum_{j=0}^{\alpha} \frac{(\alpha+1) \Gamma(j-i t \alpha)}{(\alpha+1-j) \Gamma(-i t \alpha) \Gamma(j+1)}\left(\frac{a_{n} u}{z}\right)^{j} \\
& +\left(-\frac{a_{n} u}{z}\right)^{1+\alpha} \frac{\Gamma(\alpha+1-i t \alpha)}{\Gamma(-i t \alpha) \Gamma(\alpha+1)}\left(\ln \left(-\frac{a_{n} u}{z}\right)+h_{0}^{\prime}\right),
\end{aligned}
$$

где $h_{0}^{\prime}=\Psi(\alpha+2)-\Psi(\alpha+1-i t \alpha), \Psi(x)=(d / d x)(\ln \Gamma(x))$ есть дигаммафункция,

$$
\begin{aligned}
\Psi(\alpha+1-i t \alpha)= & \frac{1}{\alpha-i t \alpha}+\frac{1}{\alpha-1-i t \alpha}+\cdots \\
& +\frac{1}{2-i t \alpha}+\frac{1}{1-i t \alpha}+\Psi(1-i t \alpha), \\
\Psi(\alpha+2)= & -\gamma+\sum_{j=1}^{\alpha+1} j^{-1}, \\
\Psi(1-i t \alpha)= & \bar{\Psi}(1+i t \alpha), \quad \alpha=1,2, \ldots \\
\operatorname{Re}(\Psi(1+i t \alpha))= & -\gamma+t^{2} \alpha^{2} \sum_{n=1}^{\infty} n^{-1}\left(n^{2}+t^{2} \alpha^{2}\right)^{-1}, \\
\operatorname{Im}(\Psi(1+i t \alpha))= & t \alpha \sum_{n=1}^{\infty}\left(n^{2}+t^{2} \alpha^{2}\right)^{-1},
\end{aligned}
$$

$\gamma$ - постоянная Эйлера (см. $\left[1,6.3 .7,6.3 .13\right.$, с. 84-85]). Значит, $\left|h_{0}^{\prime}\right| \leqslant$ $H<\infty$ для любого $t$ из ограниченного множества, где $\boldsymbol{H}$ - некоторая константа.

В случае целого $\alpha$ соотношение (13) нетрудными рассуждениями, аналогичными предыдушим, выводится из этого представления. Итак, (13) доказано для $u<0$ и $\alpha>1$.

Пусть $u>0$. Тогда в формуле (6) в выражении для $Q_{n}(t ; y, z)$ будем иметь $y=\max \left\{\beta_{n} u, \lambda_{n} x_{1}\right\}$. Так как

$$
\left|x_{1}\right| \leqslant C_{1}, \quad \beta_{n} \sim \frac{k^{1 / 2+\nu} n^{-\nu}}{L^{*}(k /(n C))}, \quad \lambda_{n} \sim n^{-\nu} L^{*}\left(\frac{1}{n C}\right),
$$

$L^{*}(1 /(n C)) L^{*}(k /(n C)) n^{-\delta}=o\left(k^{-3 \delta / 2}\right)$ для любого $\delta>0$, то $\lambda_{n} / \beta_{n}=$ $o\left(k^{(3 \delta-1) / 2-\nu}\right)$, т.е. $\lambda_{n} / \beta_{n} \rightarrow 0(n \rightarrow \infty)$. Поэтому найдется такое $n_{0}$, что $y=\beta_{n} u$ для $n>n_{0}$. Кроме того, $F\left(b_{n}+a_{n} x_{2}\right)-F\left(\beta_{n} u\right) \sim F\left(b_{n}+a_{n} x_{2}\right)$ 
при $n \rightarrow \infty$. Значит, если обозначить $Q_{n} \equiv Q_{n}\left(t ; \beta_{n} u, z\right)$, то

$$
Q_{n}=U_{n}^{k-1}(t ; z) \exp \left\{i \operatorname{tin} M_{n}(z, u)\right\}(1+o(1))
$$

где

$$
\begin{aligned}
U_{n}(t ; z)= & \frac{1+\alpha}{1+\alpha-i t}\left(-\frac{u a_{n}}{z}\right)^{i t \alpha} \\
& \times F_{1}\left(\alpha+1-i t \alpha,-i t \alpha ; \alpha+2-i t \alpha ; \frac{z}{a_{n} u}\right) \\
& -\left(-\frac{u a_{n}}{z}\right)^{1+\alpha} \frac{\Gamma(\alpha+1-i t \alpha) \Gamma(i t \alpha)}{\Gamma(\alpha+1)}
\end{aligned}
$$

Теперь, чтобы получить соотношение (13), остается повторить рассуждения для случая $u<0$.

Окончательно,

$$
\varphi_{n}(t) \rightarrow \varphi(t)=\exp \left\{-\frac{i t \alpha^{2} u^{2}}{2\left(\alpha^{2}-1\right)}-\frac{t^{2} \alpha^{2} u^{2}}{2\left(\alpha^{2}-1\right)}\right\}, \quad n \rightarrow \infty
$$

В общем случае $(m \geqslant 2)$ при $n \rightarrow \infty$ имеет место сходимость

$$
\begin{aligned}
\varphi_{n}(\mathbf{t}) \longrightarrow \varphi(\mathbf{t}) & =\mathbf{E}\left(\exp \left\{i \sum_{j=1}^{m} \xi\left(u_{j}\right) t_{j}\right\}\right) \\
& =\exp \left\{-\frac{i \alpha^{2}}{2\left(\alpha^{2}-1\right)} \sum_{j=1}^{m} u_{j}^{2} t_{j}-\frac{\alpha^{2}}{2\left(\alpha^{2}-1\right)}\left(\sum_{j=1}^{m} u_{j} t_{j}\right)^{2}\right\},
\end{aligned}
$$

которая доказывается индукцией по $m$, используя следуюшую формулу, выражаюшую гипергеометрическую функцию от $(m+1)$ переменных через гипергеометрическую функцию $m$ и одного переменных.

Лемма 2. Пусть $s=s_{1}+s_{2}+\cdots+s_{m}$. Тогда

$$
\begin{aligned}
& F_{1}\left(a, b_{1}, b_{2}, \ldots, b_{m}, b ; c ; x_{1}, x_{2}, \ldots, x_{m}, x\right) \\
& =\sum_{s_{1}, s_{2}, \ldots, s_{m}=0}^{\infty} \frac{(a)_{s}(c-a)_{s}}{(c-s-1)(c)_{2 s}} \\
& \quad \times \prod_{j=1}^{m} \frac{x_{j}^{s_{j}}}{s_{j} !}(b)_{s_{j}}(b)_{s} x^{s} F_{1}(a+s, b+s ; c+2 s ; x) \\
& \quad \times F_{1}\left(a+s, b_{1}+s_{1}, b_{2}+s_{2}, \ldots, b_{m}+s_{m} ; c+2 s ; x_{1}, x_{2}, \ldots, x_{m}\right),
\end{aligned}
$$

$|x|<1,\left|x_{j}\right|<1, j=1,2, \ldots, m$.

Формула (15) устанавливается путем сравнения коэффициентов разложения левой и правой частей. Она обобщает формулу 6.7.2.4 из [9, c. 415$]$. 
С л у ч а й (ii). Пусть $\alpha=1, u<0, \varepsilon_{n}(z)=-\beta_{n} u / z$. Тогда (см. [2, c. 117 , формула $2.10 .(9)]$ )

$$
\begin{aligned}
U_{n}(t, z)= & -2 \varepsilon_{n}^{2}(z) \sum_{j=1}^{\infty} \frac{(i t)_{j+2}}{j(j+2) !}\left(-\varepsilon_{n}(z)\right)^{j}+1+2 i t \varepsilon_{n}(z) \\
& +i t(i t-1) \varepsilon_{n}^{2}(z)\left(\ln \varepsilon_{n}(z)+h_{0}^{\prime}\right)
\end{aligned}
$$

где $h_{0}^{\prime}=\Psi(3)-\Psi(2-i t)$. Но $\left|h_{0}^{\prime}\right| \leqslant H<\infty$ для любого $t$ из ограниченного множества, $\boldsymbol{H}$ - подходящая константа. Кроме того,

$$
\begin{aligned}
\left|(-i t)_{j+2}\right| & =|(j+1-i t)(j-i t) \cdots(-i t)| \\
& \leqslant(j+2) !\left(1+\frac{|t|}{j+2}\right)\left(1+\frac{|t|}{j+1}\right) \cdots(1+|t|) \\
& \leqslant(j+2) !(1+|t|)^{j+2}
\end{aligned}
$$

Поэтому

$$
\begin{aligned}
\left|\sum_{j=2}^{\infty} \frac{(i t)_{j+2}}{j(j+2) !} \varepsilon_{n}^{j}(z)\right| & \leqslant(1+|t|)^{2} \sum_{j=1}^{\infty} \frac{\left((1+|t|)\left|\varepsilon_{n}(z)\right|\right)^{j}}{j} \\
& =(1+|t|)^{2} \ln \left(1-(1+|t|)\left|\varepsilon_{n}(z)\right|\right) \rightarrow 0, \quad n \rightarrow \infty,
\end{aligned}
$$

так как $\left(\mu(n) b_{n}\right)^{-2}=1 /(k \ln k) \rightarrow 0(n \rightarrow \infty)$, причем сходимость равномерна по $\left|x_{2}\right| \leqslant C_{2}<\infty$. Значит, в представлении $U_{n}(t ; z)$ можно ограничиться тремя последними слагаемыми:

$$
U_{n}(t ; z)=1-\frac{i t u}{\sqrt{k \ln k}}+\frac{i t(i t-1) u^{2}}{2 k}+o\left(\frac{1}{k}\right) .
$$

Кроме того $\left(\nu=\frac{1}{2}\right)$,

$$
M_{n}(z, u)=\frac{2 u \sqrt{k}}{n \sqrt{\ln k}}+o\left(\frac{1}{n}\right)+O\left(\frac{k^{3 / 2}}{n^{2} \sqrt{\ln k}}\right)+O\left(\frac{k^{\nu \ell+1 / 2}}{n^{\nu \ell+1} \sqrt{\ln k}}\right) .
$$

Следовательно, в силу (U2),

$$
Q_{n}(t ; y, z) \rightarrow \exp \left\{-\frac{t^{2} u^{2}}{2}-\frac{i t u^{2}}{2}\right\}, \quad n \rightarrow \infty
$$

и

$$
\varphi_{n}(t) \longrightarrow \exp \left\{-\frac{t^{2} u^{2}}{2}-\frac{i t u^{2}}{2}\right\}, \quad n \rightarrow \infty .
$$

Если $u>0$, то рассуждения проводятся аналогично случаю (i), и поэтому (16) будет иметь место и в этом случае. Для $m \geqslant 2$ доказательство сходимости конечномерных распределений случайных функций $\xi_{n}(u)$ к 
конечномерным распределениям функции $\xi(u)$ использует формулу (15) и проводится индукцией по $m$.

С л у ч а й (iii). Здесь $\xi_{n}(u)=-\infty$ для $u>\lambda_{n} X_{n}^{(1)}$. Кроме того, из (8) следует, что $U_{n}(t ; z)=1, n M_{n}(z, u) \rightarrow u$ для $u<z$, поэтому

$$
\varphi_{n}(t) \rightarrow \exp \{i t u\}, \quad n \rightarrow \infty,
$$

откуда получаем результат (iii) теоремы 1.

С л у ч а й (iv). Пусть $0<|\alpha|<1, u<\lambda_{n} x_{1}$ (здесь $\lambda_{n}=\beta_{n}$ ). Зафиксируем пару порядковых статистик $\left(X_{n}^{(1)}, X_{n}^{(k)}\right)$ и рассмотрим интеграл

$$
I_{n}(t ; y, z)=\int_{y}^{z}\left(v-\beta_{n} u\right)^{i t \alpha} v^{-i t \alpha} C(1+\alpha) v^{\alpha} d v
$$

Положим $z=b_{n}+a_{n} x_{2}, y=\beta_{n} x_{1}$ и обозначим $I_{n}=I_{n}\left(t ; \beta_{n} x_{1}, b_{n}+\right.$ $a_{n} x_{2}$ ). Тогда $I_{n}=I_{n}^{(1)}-I_{n}^{(2)}$, где

$$
\begin{aligned}
I_{n}^{(1)}= & C(1+\alpha) \int_{0}^{z}\left(v-u \beta_{n}\right)^{i t \alpha} v^{-i t \alpha+\alpha} d v \\
= & C(1+\alpha)\left(-u \beta_{n}\right)^{1+\alpha} \frac{\Gamma(1+\alpha-i t \alpha) \Gamma(-\alpha-1)}{\Gamma(-i t \alpha)} \\
& +C z^{1+\alpha} F_{1}\left(-i t \alpha,-\alpha-1 ;-\alpha ; \frac{u \beta_{n}}{z}\right) \\
I_{n}^{(2)}= & C(1+\alpha) \int_{0}^{x_{1} \beta_{n}}\left(v-u \beta_{n}\right)^{i t \alpha} v^{-i t \alpha+\alpha} d v \\
= & C(1+\alpha)\left(-u \beta_{n}\right)^{1+\alpha} \frac{\Gamma(1+\alpha-i t \alpha) \Gamma(-\alpha-1)}{\Gamma(i t \alpha)} \\
& +C\left(x_{1} \beta_{n}\right)^{1+\alpha} F_{1}\left(-i t \alpha,-\alpha-1 ;-\alpha ; \frac{u}{x_{1}}\right)
\end{aligned}
$$

Поэтому

$$
\begin{aligned}
U_{n}= & \frac{I_{n}}{F\left(b_{n}+a_{n} x_{2}\right)-F\left(x_{1} \beta_{n}\right)} \\
= & \frac{F_{1}\left(-i t \alpha,-\alpha-1 ;-\alpha ; u k^{-\nu}\left(1+x_{2} \nu k^{-1 / 2}\right)^{-1}\right)}{1-x_{1}^{1+\alpha} k^{-1}\left(1+x_{2} \nu k^{-1 / 2}\right)^{-1-\alpha}} \\
& -\frac{x_{1}^{1+\alpha}}{k\left(1+x_{2} \nu k^{-1 / 2}\right)^{1+\alpha}-x_{1}^{1+\alpha}} F_{1}\left(-i t \alpha,-\alpha-1 ;-\alpha ; \frac{u}{x_{1}}\right) \\
= & 1-i t(\alpha+1) u k^{-\nu}+x_{1}^{1+\alpha} k^{-1}\left(1-F_{1}\left(-i t \alpha,-\alpha-1 ;-\alpha ; \frac{u}{x_{1}}\right)\right) \\
& +o\left(\frac{1}{k}\right)
\end{aligned}
$$


и

$$
\begin{aligned}
& U_{n}^{k}=\exp \left\{-i t(1+\alpha) u k^{1-\nu}\right. \\
& \left.+x_{1}^{1+\alpha}\left(1-F_{1}\left(-i t \alpha,-\alpha-1 ;-\alpha ; \frac{u}{x_{1}}\right)\right)\right\}(1+o(1)) .
\end{aligned}
$$

В то же время при $n \rightarrow \infty$

$$
n M_{n}\left(b_{n}+a_{n} x_{2}, u\right)=u(1+\alpha) k^{1-\nu}+O\left(k^{2-\nu} n^{-1}\right)+O\left(\frac{k^{1-\nu+\nu \ell}}{n^{\nu \ell}}\right)
$$

В случае $-1<\alpha<0$ имеем $1-\nu<0$, а значит, $2-\nu<1$ и для $0<\alpha<1$ будет выполнено $0<1-\nu<\frac{1}{2}, 1<2-\nu<\frac{3}{2}$. Поэтому, в силу условия $(U 4)$,

$Q_{n}\left(t ; \beta_{n} x_{1}, a_{n} x_{2}+b_{n}\right) \longrightarrow \exp \left\{x_{1}^{1+\alpha}\left(1-F_{1}\left(-i t \alpha,-\alpha-1 ;-\alpha ; \frac{u}{x_{1}}\right)\right)\right\}$

Для $u>\lambda_{n} X_{n}^{(1)}, \xi_{n}(u)=-\infty$. Таким образом, при $n \rightarrow \infty$

$$
\varphi_{n}(t)=\mathbf{E}\left(\exp \left\{i t \xi_{n}(u)\right\}\right) \rightarrow(1+\alpha) \int_{u}^{\infty} x^{\alpha} \varphi_{x}(t) \exp \left\{-x^{1+\alpha}\right\} d x .
$$

Последнее завершает доказательство теоремы 1.

Доказательств о теоремы: 2. Обозначим $\Lambda_{n}(u)=$ $\exp \left\{\frac{1}{2} \xi_{n}(u)\right\}$. Согласно [6, с. 246, теорема 3.2.1] для доказательства сходимости распределений ощенок (в случае $\alpha \geqslant 1$ ) к предельному распределению достаточно показать:

(A) $\quad \mathbf{E}\left(\Lambda_{n}\left(u_{2}\right)-\Lambda_{n}\left(u_{1}\right)\right)^{2} \leqslant C_{3}\left(u_{2}-u_{1}\right)^{2} \quad$ для $n>n_{0},\left(u_{1}, u_{2}\right) \in \mathbf{R}^{2}$;

(Б) для любого $N>0$ существует такая постоянная $C_{N}$, что при $n>n_{0}$ и всех $u \in \mathbf{K} \subset R^{1}$, где $\mathbf{K}-$ компакт,

$$
\mathbf{E}\left(\Lambda_{n}(u)\right) \leqslant C_{N}|u|^{-N}
$$

Докажем (А). Используя неравенство Коши-Буняковского, условия $(U 1),(U 2)$ теоремы 1 , получим (считая, что $u_{2}>u_{1}$ ):

$$
\begin{aligned}
& \mathbf{E}\left(\Lambda_{n}\left(u_{2}\right)-\Lambda_{n}\left(u_{1}\right)\right)^{2} \\
& \quad=4 \mathbf{E}\left(\int_{u_{1} / \mu(n)}^{u_{2} / \mu(n)} \frac{\partial}{\partial t} f_{n}^{1 / 2}\left(X_{n}^{(1)}-t, \ldots, X_{n}^{(k)}-t\right) d t\right)^{2} \\
& \quad \leqslant C_{4} \frac{\left|u_{2}-u_{1}\right|}{\mu(n)}
\end{aligned}
$$




$$
\begin{aligned}
& \quad \times \mathbf{E}\left(\int_{u_{1} / \mu(n)}^{u_{2} / \mu(n)}\left(\frac{\partial}{\partial t} f_{n}\left(X_{n}^{(1)}-t, \ldots, X_{n}^{(k)}-t\right)\right)^{2}\right. \\
& \left.\times f_{n}\left(X_{n}^{(1)}-t, \ldots, X_{n}^{(k)}-t\right) d t\right) \\
& \leqslant \sup _{u_{1} / \mu(n) \leqslant t \leqslant u_{2} / \mu(n)} J_{n}(t) C_{5}\left(u_{2}-u_{1}\right)^{2} \leqslant C_{3}\left(u_{2}-u_{1}\right)^{2} .
\end{aligned}
$$

Итак, неравенство (А) установлено (в последнем соотношении надо повторить доказательство соответствующих частей теоремы 1 (i), (ii)).

Перейдем к выводу неравенства (Б). Пусть $\beta_{n}(u)=\mathbf{E}\left(\Lambda_{n}(u)-1\right)^{2}$. Из неравенства $1-x \leqslant e^{-x}$ и неравенства (17) получаем

$$
\mathbf{E}\left(\Lambda_{n}(u)\right)=1-\frac{1}{2} \beta_{n}^{2}(u) \leqslant \exp \left\{-\frac{1}{2} \beta_{n}^{2}(u)\right\} \leqslant \exp \left\{-C_{6} u^{2}\right\}
$$

для $|u| \leqslant C_{7} \sqrt{k}$ в случае $\alpha>1$ и для $|u| \leqslant C_{8} \sqrt{k \ln k}$ в случае $\alpha=1$, где $C_{7}, C_{8}$ есть любые положительные константы $\left(C_{6}\right.$ определяется по $\left.C_{7}, C_{8}\right)$.

Но если $|u|>C_{2}+(1+\alpha) \sqrt{k}$, когда $\alpha>1$, и $|u|>C_{9} \sqrt{k} \ln k$, когда $\alpha=$ 1 , где $C_{9}$ - достаточно большая константа, то $\int_{0}^{a_{n} x+b_{n}} f^{1 / 2}(v) f^{1 / 2}(v-$ $\left.\beta_{n} u\right) d v=0$ для $|x| \leqslant C_{2}$. Неравенство (Б) и, значит, результаты (i), (ii) теоремы 2 следуют из (18). Результат (iii) теоремы 2 следует из теоремы 5.3.1 [6, с. 348].

Доказательст в о те о рем ы 3 повторяет доказательство теорем 2.12 .1 и 5.5 .3 из [6, с. 223-224, с. 372], поэтому опушено.

5. Примеры. Распределениями, удовлетворяющими условию (1) (или (2)), являются $\left(\alpha>-1, \ell>0, D_{1}>0\right.$ и $C>0$ известны):

1) распределение Вейбулла с плотностью

$$
f(x)=C(1+\alpha) x^{\alpha} \exp \left\{-x^{1+\alpha}\right\}, \quad x>0
$$

2) гамма-распределение с плотностью

$$
f(x)=C(1+\alpha) x^{\alpha} \exp \left\{-D_{1} x^{\ell}\right\}, \quad x>0
$$

3) бета-распределение с плотностью

$$
f(x)=C(1+\alpha) x^{\alpha}\left(1-D_{1} x^{\ell}\right)^{\rho}, \quad 0<x<1, \quad \rho>0
$$

4) бета-распределение 2-го рода с плотностью

$$
f(x)=C x^{\alpha}\left(1+D_{1} x^{\ell}\right)^{-q}, \quad q>0
$$

где $C$ - нормирующая константа.

Для этих примеров соответствующая функция распределения $F(x)$ может быть записана в виде $F(x)=R(x)=C x^{1+\alpha}\left(1+D_{1} x^{\ell}+o\left(x^{\ell}\right)\right)$.

4 Теория вероятностей и ее применения, № 3 
Асимптотически взаимно обратной к ней функцией при $\dot{x} \downarrow 0$ будет $R^{*}(x)=(x / C)^{\nu}\left(1-\nu D_{1}(x / C)^{\ell \nu}+o\left(x^{\ell \nu}\right)\right)$.

Пусть

$$
f(x)=(1+\alpha) x^{\alpha}, \quad 0<x<1
$$

Для $0<2 \nu<1,2 \nu=1$ и $\nu=1$ (соответственно, для $1<\alpha<\infty$, $\alpha=1, \alpha=0$ ) приведем простые оценки параметра сдвига $\theta$ плотности (19), представляющие собой линейные комбинации порядковых статистик $X_{n}^{(1)}, X_{n}^{(2)}, \ldots, X_{n}^{(k)}$.

Определим статистику $t_{n}^{*}$ равенствами

$$
\begin{aligned}
t_{n}^{*} & =t_{n}^{*}\left(X_{n}^{(1)}, \ldots, X_{n}^{(k)}\right) \\
& =(1-2 \nu)(1-\nu)^{-1}\left(\nu k^{2 \nu-1} \sum_{j=1}^{k} j^{-2 \nu} X_{n}^{(j)}+X_{n}^{(k)}\right)
\end{aligned}
$$

в случае $2 \nu<1$,

$$
t_{n}^{*}=(\ln k)^{-1} \sum_{j=1}^{k} j^{-1} X_{n}^{(j)}
$$

в случае $2 \nu=1$,

$$
t_{n}^{*}=X_{n}^{(1)}+\frac{X_{n}^{(k)}-X_{n}^{(1)}-1}{n-k+2}
$$

в случае $\nu=1$.

ПІокажем сначала, что $t_{n}^{*}\left(X_{n}^{(1)}-\theta, \ldots, X_{n}^{(k)}-\theta\right)=t_{n}^{*}\left(X_{n}^{(1)}, \ldots, X_{n}^{(k)}\right)-$ $\theta+o(1), \theta \in \mathbf{R}^{1}$, при $n \rightarrow \infty$. Для этого воспользуемся формулой 23.2.9 из $[1$, с. 610$]$ :

$$
\begin{gathered}
\sum_{j=1}^{k} j^{-s}=\zeta(s)+(1-s)^{-1} k^{1-s}+s \int_{k}^{\infty}\{x\} x^{-1-s} d x \\
k=1,2, \ldots ; \quad \operatorname{Re} s>0
\end{gathered}
$$

когда $s=2 \nu<1$, где $\zeta(s)$ - дзета-функция Римана, а $\{x\}-$ дробная часть числа $x$. Отметим, что функция $\zeta(s)$ регулярна для комплексных значений $s \neq 1$ (при $s=1$ она имеет простой полюс с вычетом 1). Так как $\{x\} \leqslant 1$, то из (23) заключаем, что при $k \rightarrow \infty$

$$
\sum_{j=1}^{k} j^{-2 \nu}=\zeta(2 \nu)+(1-2 \nu)^{-1} k^{1-2 \nu}+O\left(k^{-2 \nu}\right)
$$


Если $2 \nu=1$, то (см. [5, с. 16, формула (0.131)])

$$
\begin{aligned}
\sum_{j=1}^{k} j^{-1} & =\gamma+\ln k+(2 k)^{-1}-\sum_{j=2}^{\infty} \frac{\Gamma(k) A_{j}}{\Gamma(k+j)} \\
A_{j} & =j^{-1} \int_{0}^{1} x(1-x)(2-x) \cdots(j-1-x) d x,
\end{aligned}
$$

откуда следует, что $0<A_{j} \leqslant \Gamma(j-1), j=2,3, \ldots$, и

$$
\sum_{j=2}^{\infty} \frac{\Gamma(k) A_{j}}{\Gamma(k+j)} \leqslant \Gamma(k) \sum_{j=1}^{\infty} \frac{\Gamma(j)}{\Gamma(k+1+j)} .
$$

По формуле 5.2.11.10 из [8, с. 710$]$

$$
\Gamma(k) \sum_{j=1}^{\infty} \frac{\Gamma(j)}{\Gamma(k+1+j)}=k^{-2},
$$

и, значит,

$$
\sum_{j=1}^{k} j^{-1}=\gamma+\ln k+(2 k)^{-1}+O\left(k^{-2}\right), \quad k \rightarrow \infty .
$$

Из (24) и (25) получаем, что если $2 \nu<1$, то

$$
\begin{aligned}
& (1-2 \nu)(1-\nu)^{-1}\left(\nu k^{2 \nu-1} \sum_{j=1}^{k} j^{-2 \nu}+1\right) \\
& =1+\nu(1-2 \nu) \zeta(2 \nu)(1-\nu)^{-1} k^{2 \nu-1}+O\left(k^{-1}\right),
\end{aligned}
$$

а если $2 \nu=1$, то

$$
(\ln k)^{-1} \sum_{j=1}^{k} j^{-1}=1+\gamma(\ln k)^{-1}+O\left((k \ln k)^{-1}\right) .
$$

Таким образом,

$$
t_{n}^{*}\left(X_{n}^{(1)}-\theta, \ldots, X_{n}^{(k)}-\theta\right)=t_{n}^{*}\left(X_{n}^{(1)}, \ldots, X_{n}^{(k)}\right)-\theta+O\left(k^{2 \nu-1}\right),
$$

если $2 \nu<1$ и

$$
t_{n}^{*}\left(X_{n}^{(1)}-\theta, \ldots, X_{n}^{(k)}-\theta\right)=t_{n}^{*}\left(X_{n}^{(1)}, \ldots, X_{n}^{(k)}\right)-\theta+O\left((\ln k)^{-1}\right),
$$

если $2 \nu=1$.

Для $\nu=1$ статистика (22) есть оценка Питмена параметра сдвига $\theta$ плотности (19), поэтому $t_{n}^{*}\left(X_{n}^{(1)}-\theta, \ldots, X_{n}^{(k)}-\theta\right)=t_{n}^{*}\left(X_{n}^{(1)}, \ldots, X_{n}^{(k)}\right)-\theta$. 
Введем обозначение:

$$
c_{n}= \begin{cases}n^{-\nu}(1-2 \nu)(1-\nu)^{-1}\left(\nu k^{2 \nu-1} \sum_{j=1}^{k} j^{-\nu}+k^{\nu}\right), & \text { если } 2 \nu<1, \\ n^{-1 / 2}(2 \ln k)^{-1} \sum_{j=1}^{k} j^{-1 / 2}, & \text { если } 2 \nu=1, \\ 0, & \text { если } \nu=1 .\end{cases}
$$

Теорема 4. Пусть $2 \nu \leqslant 1$ или $\nu=1$,

$$
T_{n}=t_{n}^{*}-c_{n}
$$

есть оченка параметра сдвига $\theta$ плотности $f(x-\theta)$, где $f(x)$ определяется формулой (19), $t_{n}^{*}$ - формулами (20)-(22), а с (26)-(28) соответственно.

Тогда

(i) ecлu $2 \nu<1, m o$

$$
(1+\alpha) n^{\nu} k^{1 / 2-\nu}\left(T_{n}-\theta\right) \underset{n \rightarrow \infty}{\stackrel{d}{\longrightarrow}} N\left(0,(1-2 \nu) \nu^{2}(1-\nu)^{-2}\right)
$$

(ii) ecлu $2 \nu=1, m o(n \ln k)^{1 / 2}\left(T_{n}-\theta\right) \underset{n \rightarrow \infty}{\stackrel{d}{\longrightarrow}} N(0,1)$;

(iii) ecлu $\nu=1$, mo $n\left(T_{n}-\theta\right) \underset{n \rightarrow \infty}{\stackrel{d}{\longrightarrow}} \eta-1$.

Д ок а 3 а те ль с т в о. Рассмотрим пункты (i), (ii) теоремы 4 и получим сначала главные члены дисперсии оценки (29) параметра $\theta$. Для этого воспользуемся следуюшим представлением из работы [5] для порядковых статистик из распределения (19):

$$
X_{n}^{(j)}=\left(\sum_{i=1}^{j} \frac{\eta_{i}}{n}\right)^{\nu}+O\left(\left(\frac{j}{n}\right)^{\nu+1}\right) \quad(n \rightarrow \infty)
$$

равномерно по $1 \leqslant j \leqslant k$, где $\eta_{1}, \eta_{2}, \ldots, \eta_{n}$ - независимые стандартные показательно распределенные случайные величины.

Положим

$$
Z_{j}=\sum_{i=1}^{j}\left(\eta_{i}-1\right), \quad 1 \leqslant j \leqslant k .
$$

Тогда (см. $[5$, с. 561$])$

$$
\left(\sum_{i=1}^{k} \eta_{i}\right)^{\nu}=k^{\nu}+\nu Z_{k} k^{\nu-1}+O\left(k^{\nu-1} \ln \ln k\right) .
$$


Поэтому

$$
\begin{aligned}
\sum_{j=1}^{k-1} j^{-2 \nu}\left(\sum_{i=1}^{j} \eta_{i}\right)^{\nu} & =(1-\nu)^{-1} k^{1-\nu}-\nu \sum_{j=1}^{k} j^{-1-\nu} Z_{j}+O(1) \\
\sum_{j=1}^{k} j^{-1-\nu} Z_{j} & =\sum_{i=1}^{k}\left(\eta_{i}-1\right) \sum_{j=i}^{k} j^{-1-\nu} \\
\mathbf{D}\left(\sum_{j=1}^{k} j^{-1-\nu} Z_{j}\right) & =\sum_{j=1}^{k}\left(\sum_{j=i}^{k} j^{-1-\nu}\right)^{2}=S_{k}(\nu)
\end{aligned}
$$

Ho

$$
\begin{aligned}
S_{k}(\nu) & =\sum_{i=1}^{k} \sum_{j=i}^{k} \sum_{h=i}^{k}(j h)^{-1-\nu}=\sum_{j=1}^{k}\left(\sum_{h=1}^{j} \sum_{i=1}^{h}+\sum_{h=j+1}^{k} \sum_{i=1}^{j}\right)(j h)^{-1-\nu} \\
& =\sum_{j=1}^{k} j^{-1-\nu} \sum_{h=1}^{j} h^{\nu}+\sum_{j=1}^{k} j^{\nu} \sum_{h=j+1}^{k} h^{-1-\nu} \\
& =\sum_{j=1}^{k} j^{-1-\nu} \sum_{h=1}^{j} h^{-\nu}+\sum_{j=1}^{k} j^{-\nu} \sum_{h=1}^{k} h^{-1-\nu}-\sum_{j=1}^{k} j^{-1-2 \nu} \\
& =2 \sum_{j=1}^{k} j^{-1-\nu} \sum_{i=1}^{j} i^{-\nu}-\sum_{j=1}^{k} j^{-1-2 \nu} .
\end{aligned}
$$

Таким образом, получаем:

$$
D\left(\sum_{j=1}^{k} j^{-1-\nu} Z_{j}\right)=2 \sum_{j=1}^{k} j^{-1-\nu} \sum_{i=1}^{j} i^{-\nu}-\sum_{j=1}^{k} j^{-1-2 \nu} .
$$

Для $2 \nu \leqslant 1$ ряд $\sum_{j=1}^{\infty} j^{-1-2 \nu}$ сходится, поэтому при $k \rightarrow \infty$

$$
S_{k}(\nu) \sim \begin{cases}4 \ln k, & \text { если } \quad \nu=\frac{1}{2}, \\ 2 k^{1-2 \nu} /(1-\nu)(1-2 \nu), & \text { если } \nu<\frac{1}{2} .\end{cases}
$$

Для $\nu>\frac{1}{2}$ ряд $S_{k}(\nu)$ сходится при $k \rightarrow \infty$. Это следует из монотонности и ограниченности последовательности $\left\{S_{k}(\nu)\right\}, k=1,2, \ldots$. Действительно, для $\frac{1}{2}<\nu<1$, выполняется неравенство $S_{k}(\nu) \leqslant 2(1-$ $\nu)^{-1} \zeta(2 \nu)-\zeta(1+2 \nu)$; а для $\nu>1$ верно неравенство $S_{k}(\nu) \leqslant 2 \zeta(1+$ $2 \nu)$. Для $\nu=1$ сумма $S_{k}(1)$ не превосходит сходяшегося ряда $2 \zeta(2)+$ $\sum_{j=2}^{\infty} j^{-2} \ln j$. Положим $\lim _{k \rightarrow \infty} S_{k}(\nu)=\varkappa(\nu)$.

Приведем некоторые компьютерные значения $\boldsymbol{x}(\nu): \mathfrak{x}(0.85)=5.111$, $\varkappa(0.9)=4.477, x(0.95)=3.988, \varkappa(1.0)=3.602, \varkappa(1.1)=3.034, x(1.2)=$ $2.640, x(1.5)=1.967, x(2.0)=1.495, \varkappa(4.0)=1.077, \varkappa(8.0)=1.004$. Кроме 
того, $S_{10000}(0.55)=21.116, S_{100000}(0.55)=23.385, S_{10000}(0.60)=14.987$, $S_{100000}(0.6)=15.824, S_{10000}(0.75)=7.108, S_{100000}(0.75)=7.167$.

Заметим также, что для $\nu=\frac{1}{2}$ последовательность $a_{k}=S_{k}(\nu) \times$ $(4 \ln k)^{-1}$ при $k \rightarrow \infty$ сходится к 1 при $k \rightarrow \infty$, но довольно. медленно. Tak, $a_{1000}=0.81, a_{50000}=0.876$.

Из вышесказанного следует, что если $\nu<\frac{1}{2}$, то

$$
\begin{aligned}
\mathbf{D}\left(T_{n}\right) & \sim n^{-2 \nu} \nu^{2}(1-2 \nu)^{2}(1-\nu)^{-2} k^{4 \nu-2} \sum_{i=1}^{k}\left(\sum_{j=i}^{k} j^{-1-\nu}+k^{-\nu}\right)^{2} \\
& \sim n^{-2 \nu} \nu^{2}(1-2 \nu)(1-\nu)^{-2} k^{2 \nu-1}
\end{aligned}
$$

а если $\nu=\frac{1}{2}$, то

$$
\mathrm{D}\left(T_{n}\right) \sim(4 n)^{-1}(\ln k)^{-2} \sum_{i=1}^{k}\left(\sum_{j=i}^{k} j^{-3 / 2}\right)^{2} \sim(n \ln k)^{-1} .
$$

Воспользуемся теперь следуюшей теоремой 5 (см. [3], с. 198). Пусть $Y_{1}, Y_{2}, \ldots$ - независимые одинаково распределенные случайные величины с конечным математическим ожиданием $\mu$ и конечной дисперсией $\sigma^{2}$. В таком случае статистика $T_{a}=\sum_{i=1}^{N} a_{i} Y_{i}$, где $\left(a_{1}, a_{2}, \ldots, a_{N}\right)-$ ненулевой действительный вектор, при условии

$$
\sum_{i=1}^{N} \frac{a_{i}^{2}}{\max _{1 \leqslant i \leqslant N} a_{i}^{2}} \longrightarrow \infty \quad(N \rightarrow \infty)
$$

асимптотически нормальна с параметрами

$$
\mu_{a}=\mu \sum_{i=1}^{N} a_{i}, \quad \sigma_{a}^{2}=\sigma^{2} \sum_{i=1}^{N} a_{i}^{2} .
$$

Так как для $2 \nu \leqslant 1$ ряд $\sum_{j=1}^{\infty} j^{-2 \nu}$ расходится, а

$$
\max _{1 \leqslant i \leqslant k}\left(\sum_{j=i}^{k} j^{-1-\nu}\right)^{2} \leqslant C_{9} \zeta^{2}(1+\nu),
$$

то из равенств $(20),(30)-(34)$ и теоремы 5 , примененной к статистикам $T_{n}$ заключаем, что в случае $2 \nu<1$ предельное распределеніие последовательности $\mu(n)\left(T_{n}-\theta\right)$ будет нормальным $N\left(0,(1-2 \nu) \nu^{2}(1-\nu)^{-2}\right)$, где $\mu(n)=\nu^{-1} n^{\nu} k^{1 / 2-\nu}$, а если $2 \nu=1$, то предельное распределение $(n \ln k)^{1 / 2}\left(T_{n}-\theta\right)$ - нормальное $N(0,1)$.

Рассмотрим пункт (iii). Для $\nu=1$ прямые вычисления дают следуюшее значение дисперсии

$$
\mathbf{D}\left(T_{n}\right)=\frac{n-k+1}{(n-k+2)(n+1)(n+2)},
$$


Значит,

$$
D\left(T_{n}\right) \sim n^{-2}, \quad n \rightarrow \infty .
$$

Из леммы 1 (см. также [7, с. 34-35]) следует, что

$$
n X_{n}^{(1)} \underset{n \rightarrow \infty}{\stackrel{d}{\longrightarrow}} \eta, \quad \text { a } \quad X_{n}^{(k)} \underset{n \rightarrow \infty}{\stackrel{p}{\rightarrow}} 0 .
$$

Это доказывает утверждение (iii) и завершает доказательство теоремы 4.

Для $\frac{1}{2}<\nu<1$ и $\nu>1$ (т.е. для $0<\alpha<1$ и $-1<\alpha<0$ соответственно) можно предложить две оценки параметра $\theta$ :

$$
\bar{t}_{n}=\frac{\sum_{j=1}^{k} j^{-2 \nu} X_{n}^{(j)}-\sum_{j=1}^{k} j^{-\nu}}{\zeta(2 \nu)}
$$

и

$$
\widehat{T}_{n}=X_{n}^{(1)}-\omega(\nu) n^{-\nu}, \quad \text { где } \omega(\nu)=\nu^{-\nu} \Gamma(1+\nu) .
$$

Оценка (35) (а также (20) и (21)) предложена, по-видимому, впервые. Оценка (36) рассматривалась в $[11$, с. 47 , формулы (74), (75)] и в $[6$, c. 420-421]. Оценки (35) и (36) асимптотически несмешены, и

$$
\begin{aligned}
& R_{1}(\nu)=\lim _{n \rightarrow \infty} \mathbf{E}_{\theta}\left(n^{\nu}\left(\vec{t}_{n}-\theta\right)\right)^{2}=\nu^{2} \varkappa(\nu) \zeta^{-2}(2 \nu), \\
& R_{2}(\nu)=\lim _{n \rightarrow \infty} \mathbf{E}_{\theta}\left(n^{\nu}\left(\widehat{T}_{n}-\theta\right)\right)^{2}=\nu^{-2 \nu}\left(\Gamma(1+2 \nu)-\Gamma^{2}(1+\nu)\right) .
\end{aligned}
$$

Заметим, что для плотности (19) можно показать, что дисперсия оценки Питмена (как и дисперсии статистик (35) и (36)) имеет порядок $n^{-2 \nu}$. Численные значения величин $R_{1}(\nu)$ и $R_{2}(\nu)$, рассчитанные по таблицам $12-14$ из $[20$, с. 58,59$]$ и по таблице 26 из [20, с. 92-93], приведены в таблище 1.

Таблица 1: Предельные квадратичные риски для $n^{\nu}\left(\bar{t}_{n}-\theta\right)$ и $n^{\nu}\left(\widehat{T}_{n}-\theta\right)$

\begin{tabular}{|c|c|c|c|c|c|c|c|}
\hline$\nu$ & 0.600 & 0.800 & 0.850 & 0.900 & 1.000 & 2.000 & 4.000 \\
\hline$R_{1}(\nu)$ & 0.182 & 0.732 & 0.875 & 1.024 & 1.331 & 5.104 & 17.088 \\
\hline$R_{2}(\nu)$ & 0.560 & 0.803 & 0.858 & 0.908 & 1.000 & 1.250 & 0.606 \\
\hline
\end{tabular}

Таблица 1 показывает, что при $\nu$, меньших примерно 0.83 (соответствуюшее значение $\alpha$ равно 0.205 ) оценка $\bar{t}_{n}$ лучше, чем $\widehat{T}_{n}$, а при $\nu$, больших 0.85 , оценка $\widehat{T}_{n}$ лучше, чем $\bar{t}_{n}$. Так как $R_{2}(\nu) \sim(\pi / \nu)^{1 / 2}(2 / e)^{2 \nu}$, то $R_{2}(\nu) \rightarrow 0$ при $\nu \rightarrow \infty$ (т.е. когда $\alpha \rightarrow-1$ ). Кроме того, $\varkappa(\nu) \rightarrow 1$, $\zeta(2 \nu) \rightarrow 1$ при $\nu \rightarrow \infty$. Поэтому $R_{1}(\nu) \sim \nu^{2}$, когда $\nu \rightarrow \infty$. Если $\nu \rightarrow \frac{1}{2}$, 
то $R_{1}(\nu) \sim \nu^{2}(2 \nu-1)^{2}$, т.е. $R_{1}(\nu) \rightarrow 0$, в то же время $R_{2}\left(\frac{1}{2}\right)=(4-\pi) / 8 \approx$ 0.107 .

\section{СПИСОК ЛИТЕРАТУРЫ}

1. Абрамович М., Стигая И. Справочник по специальным функциям. М.: Наука, 1979, $832 \mathrm{c}$.

2. Бейтмен $Г$., Эрдейи А. Высшие трансцендентные функции. Т. 1; 2. М.: Наука, 1973,299 c.; 1974, $296 \mathrm{c}$.

3. Гаек Я., Шидак 3. Теория ранговых критериев. М.: Наука, 1971, 376 с.

4. Гихман И. И., Скороход А. В. Введение в теорию случайных процессов. М.: Наука, 1965, $656 \mathrm{c}$.

5. Градитейя И. С., Рыжик И. М. Таблицы интегралов, сумм, рядов и произведений. М.: ГИФМЛ, 1963, $1100 \mathrm{c}$.

6. Ибрагимов И. А., Хасьминский $P$. З. Асимптотическая теория оценивания. М.: Наука, 1979, $528 \mathrm{c}$.

7. Лидбеттер $M$. ., Ротсен $X$., Лиядерен $Г$. Экстремумы случайных последовательностей и процессов. - М.: Мир, 1989, $392 \mathrm{c.}$

8. Прудкиков А. И., Брычков Ю. А., Маричев О. И. Интегралы и ряды: элементарные функции. М.: Наука, 1981, 800 c.

9. Прудников А. И., Брычков Ю. А., Маричев О. И. Интегралы и ряды: дополнительные главы. М.: Наука, 1986, 800 с.

10. Сенета $E$. Правильно меняющиеся функции. М.: Наука, 1985, 832 с.

11. Смирнов $H$. В. Теория вероятностей и математическая статистика: избранные труды. М.: Наука, 1970, 289 с.

12. Тихов $M$. С. Асимптотический анализ статистических оценок по цензурированным центральным и промежуточным членами вариационного ряда выборкам. Записки научн. семин. ЛОМИ, 1981, т. 108, с. 170-192.

13. Тихов $M$. С. Оценки параметров нерегулярных в окрестности квантилей плотностей по отрезкам вариационного ряда. - Теория вероятн. и ее примен., 1987, т. 32 , в. 2, с. $382-387$.

14. Тихов $M$. C. Асимптотики отношения правдоподобия по отрезкам вариационного ряда. - Известия ВУЗов: Математика, 1990, № 7, с. 69-79.

15. Тихов̈ $M$. C. Асимптотики T-оценок. - Теория вероятн. и ее примен., 1992, т. 37, B. 4 , с. $658-675$.

16. Яяке E., Эмде Ф., Леш Ф. Специальные функции. М.: Наука, 1968, 344 с.

17. Csörgö $S$. Limit theorems for sums of order sțatistics. - В сб.: 6 Международная летняя школа по теории вероятностей и математической статистике (Варна, 1988). Sofia, 1989 , c. 5-37.

18. Davis R., Resnik $S$. Tail estimates motivated by extreme value theory. - Ann. Statist., 1984, v. 12, p. 1467-1487.

19. Hall $P$. On estimating the endpoint of a distribution. - Ann. Statist., 1982, v. 10, p. 556-568.

20. Hall $P$., Welsh A. $H$. Adaptive estimates of parameters of regular variation. - Ann. Statist., 1985, v. 13, p. 331-341.

21. Rényi $A$. On theory of order statistics. - Acta Math. Acad. Sci. Hung., 1953, v. 4, p. 191-232. 